\title{
A Segmentation Method for Mammogram X-ray Image Based on Image Enhancement with Wavelet Fusion
}

\author{
Liang Zhengyou ${ }^{1,}$, , Gou Xiaoshan ${ }^{2, b}$ \\ ${ }^{1}$ College of computer and electronics information, Guangxi University, Nanning, Guangxi, 30004, \\ China \\ ${ }^{2}$ College of information science and technology, Chengdu University, Chengdu, Sichuan, 610106, \\ China \\ azhyliang@gxu.edu.cn, b530061041@qq.com
}

Keywords: Breast X-ray image; Wavelet transform; Image enhancement; Image segmentation; Live-wire interactive segmentation algorithm

\begin{abstract}
A segmentation method based on image enhancement method with wavelet fusion was proposed to segment X-ray mammogram images with low pixel and unclarity. Firstly, a breast X-ray image was pretreated by histogram equalization and median filtering. Then a wavelet fusion algorithm with the rule of taking large low frequency energy and high-frequency variance was applied to the pretreated image, subsequently producing an enhanced image. Secondly, an improved live-wire interactive segmentation algorithm with qualification to make the shortest path search was used to divide suspicious masses on the enhanced image. Related experiments were carried out on typical data-sources that came from the Mammographic Image Analysis Society mini-database and clinical mammography images, and the results indicated that the algorithm have good effect on image enhancement and segmentation.
\end{abstract}

\section{Introduction}

Image enhancement and image segmentation are important in the detection of breast lumps. X-ray Mammography is one of the most commonly used and most effective breast cancer screenings so that there are many researches in X-ray mammogram image segmentation. Pereira[1] proposed an algorithm for segmentation and detection of breast cancer. In their algorithm, an artifact removal algorithm is firstly implemented by an image denoising and gray-level enhancement method based on wavelet transform and Wiener filter. Then a method for detection and segmentation of masses is employed in mammograms, which uses multiple thresholding, wavelet transform and genetic algorithm. YANG[2] uses an immune algorithm-based detector to find the region of interest (ROI) and reduce the non-object area of ROI, which accords to the features of lumps such as local high intensity and local uniformity. Furthermore, C-V level set model is employed to segment the RO Immure precisely by automatically setting the initial level. The method improves the performance and the extent of segment automation. LI[3] uses spectral clustering combined with edge information to segment the pectoral muscle. She proposed a region merging algorithm on the basis of the intensity distribution of regions and the characteristic of triangle shape, and the region merging algorithm is used to identify the pectoral muscle. Cao, A.Z[4] developed a breast mass segmentation scheme, which is based on the concept of deterministic annealing and incorporated with a gain adaptive contrast enhancement (GACE) preprocessing technique. The probability distribution of gray levels within the region of interest after GACE filter is estimated and a series of image partitions obtained according to the intensity proximity. Amutha[5] uses mathematical morphology and improved double orthogonal wavelet transform technology to enhance image of breast by distinguishing the edge pixels in noise area. Aydin[6] studied an enhancement image technology for breast microcalcifications images based on neural network technology and wavelet transform. Luan [7] enhances breast X-ray image by wavelet coefficients weighted fusion. Sakellaropoulos[8] uses adaptive 2D wavelet transform to improve breast $\mathrm{X}$-ray image contrast and reduce image noise. 
Although many progresses have been made in X-ray Mammogram Image Segmentation, due to the lack of contrast and more image noise of breast X-ray image of breast, more researches need to be done to overcome the lack of contract and image noise. In this paper, a segmentation method based on image enhancement method with wavelet fusion is presented. The algorithm is based on the image preprocessing with histogram equalization method and median filter, and an efficient fusion method with the rule of taking large low frequency energy and high-frequency variance was applied to the pretreated image, the results show that this method can improve the image contrast, restrain noise and keep the edge details. Furthermore, an improved live-wire interactive segmentation algorithm ${ }^{[10]}$ is used to edge division of suspicious masses. The experimental results indicate that the proposed method can reduce noise response, and accurately locate actual edge to guarantee edge's continuity with better segmentation.

\section{Image enhancements and segmentation technology}

In this section, some technologies of image enhancement and segmentation were introduced.

Histogram equalization method. Histogram equalization method changes the histogram of original image into uniform distribution. The input image is converted into having the same number of gray levels on each pixel point. By expanding gray level of the input image, the image contrast is improved.

Image denoising. It is needed to denoise in treatment of breast images, because breast tissue has its special nature and consequently breast X-ray contains more noise. Median filter is a nonlinear signal processing way to reduce image noise, which bases on sort statistical theory. In median filter, the value of one bit is substituted by the middle value of the points on its nearby field. Median filter is a field operational in digital image processing. It sorts pixels by gray level, and then selects intermediate values as output pixel values.

Improved live-wire segmentation algorithm. Live-wire segmentation is a classic interactive segmentation tool, which is based on dynamic boundary planning proposed by Barrett and Mortensen[9]. It is efficient, accurate and reproducible for boundary extraction $[9,10]$.

Improved live-wire segmentation algorithm. The core idea of Live-wire algorithm includes three steps ${ }^{[9,10]}$ : Firstly, the image is formulated as a directed graph. Each pixel of the image is represented as a node of the Directed graph, and an edge between adjacent pixels as an edge of the Directed graph. Secondly, a cost function is defined by the figure gradient information for each side. Cost function is given a smaller value for the generation of strong edges and a greater value for non-strong edge. Meanwhile, assign 0 to the arc between adjacent pixels, and assign $+\infty$ to the arc for non-contiguous pixels. Thus, the problem of finding border is turned into the optimal path problem between start node and target node. Lastly, the optimal path search algorithm is used to find the shortest path between two points, which is regarded as the boundary of the object. An improving algorithm for the live-wire segmentation algorithm was proposed in Paper [10]. In the paper, Dang ${ }^{[10]}$ presents that Canny operator instead of Laplacian operator is used to construct the function, as Eq. 3. It does as precisely as possible to locate the actual edge while reducing the noise, and ensuring the continuity of the edge.

$$
l_{\text {new }}(p, q)=\omega_{G} \cdot f_{G}(q)+\omega_{C} \cdot f_{C}(q)+\omega_{D} \cdot f_{D}(p, q)
$$

In Eq. 3, $f_{C}(q)$ is the feature for Canny operator, $\omega_{C}$ is its weights.

$$
f_{C}(q)=\left\{\begin{array}{llll}
0 & q & \text { is edge } & \text { dot } \\
1 & q \text { isn't edge dot }
\end{array}\right.
$$

Eq. 4 is the expression of edge detection feature function for Canny.

The gradient change named $G$ is used as a number function in an inverse linear ramp function. The inverse linear ramp function is shown as Eq. 5. 


$$
f_{G}(q)=1-\frac{G(q)}{\max (G)}
$$

$G(q)$ is the mean gradient magnitude at pixel point $q, \max (G)$ the mean maximum gradient magnitude in an image.

Gradient direction adds a smoothness constraint to the boundary by associating a high cost with sharp changes in boundary direction. Letting $D(p)$ be the unit vector which is normal to gradient direction at point $p$, the formulation of gradient direction feature cost is:

$$
f_{D}(p, q)=\frac{2}{3 \pi}\left\{\cos \left[d_{p}(p, q)\right]^{-1}+\cos \left[d_{q}(p, q)\right]^{-1}\right\}
$$

Where, $d_{p}(p, q)$ and $d_{q}(p, q)$ are defined as Eq. 7 and Eq. 8 respectively.

$$
\begin{aligned}
& d_{p}(p, q)=D(p) \bullet L(p, q) \\
& d_{q}(p, q)=L(p, q) \bullet D(q)
\end{aligned}
$$

$L(p, q)$ is defined as Eq. 9. It is the normalized bidirectional link between pixels $p$ and $q$, and returns a direction value. The direction value of the link between $p$ and $q$ is minimized, and $0 \leq l(p, q) \leq 1$.

$$
L(p, q)=\left\{\begin{array}{lll}
q-p & \text { if } & D(p) \bullet(q-p) \geq 0 \\
p-q & \text { if } & D(p) \bullet(q-p)<0
\end{array}\right.
$$

Improved shortest path search strategy. The traditional live-wire segmentation algorithm uses Dijkstra algorithm to get the shortest path. However, it traverses all nodes in the image, which makes low efficiency. Wang ${ }^{[11]}$ presents a new path search strategy with restrictions in searching the shortest path from starting point to destination point. It reduces the number of nodes in the search, improves the searching efficiently. Its idea is to begin from the start point, and search for optimal path from near to far. The minimal weight accumulation node is selected form search points in each search. The node's accumulated value and the right path direction is updated and put into the search collection when a new node in its four areas of a new optimal path occurs. After the target is added to the collection point, the trail search is stopped to find out the optimal path reverse direction. Details can be seen in Paper [11].

\section{A new image segmentation method design}

Image enhancement based on wavelet fusion. Wavelet transform can be used to analyse time-domain and frequency domain. Fusion algorithm based on wavelet transform reduces the correlation between layers, and gets better fusion results.

The basic principle based on wavelet transform is described as follows: for one image , it gets $(3 \mathrm{~L}+1)$-level sub-band when decomposed form L-level wavelet, which with one $C_{i}$ in low frequency and 3L in high frequency as $D_{h}, D_{v}$ and $D_{d}$. the original image is on behalf by $f(x, y)$, noted as $C_{0}$. Scale coefficients $\Phi(x)$ and wavelet coefficients $\Psi(x)$ corresponding filter coefficient matrix are set as $H$ and $G$. Two-dimensional wavelet decomposition algorithm can be described as:

$$
\left\{\begin{array}{l}
C_{i+1}=H C_{i} H^{\prime} \\
D_{i+1}^{h}=G C_{i} H^{\prime} \\
D_{i+1}^{v}=H C_{i} G^{\prime} \\
D_{i+1}^{d}=G C_{i} G^{\prime}
\end{array}\right.
$$


In Eq. 10, $i$ represents decomposition level, $h$ represents horizontal component, $v$ represents vertical component and $d$ represents diagonal component. $H^{\prime}$ and $G^{\prime}$ are conjugate transposed matrixes.

The distribution of breast X-ray image gray is not uniform. The main information is concentrated in the low-frequency part. The high-frequency details are not rich, with more noise. So the decomposition layer number that we choose is two-level in this paper. Firstly, Histogram equalization and Median filter algorithm are used in image pretreatment respectively. Secondly, the pretreated images are decomposed by Wavelet transform. Lastly, multi-scale images are merged by fusion algorithm based on the rules of selecting the biggest energy in low frequency and the largest variance in high-frequency, and a multiple enhanced image is created. Breast X-ray images are decomposed at layer 2 by wavelet transfer. It contains seven different bands after decomposition, which includes a low-frequency sub-band and six high-frequency sub-bands. The basic procedure is shown as Fig. 1.

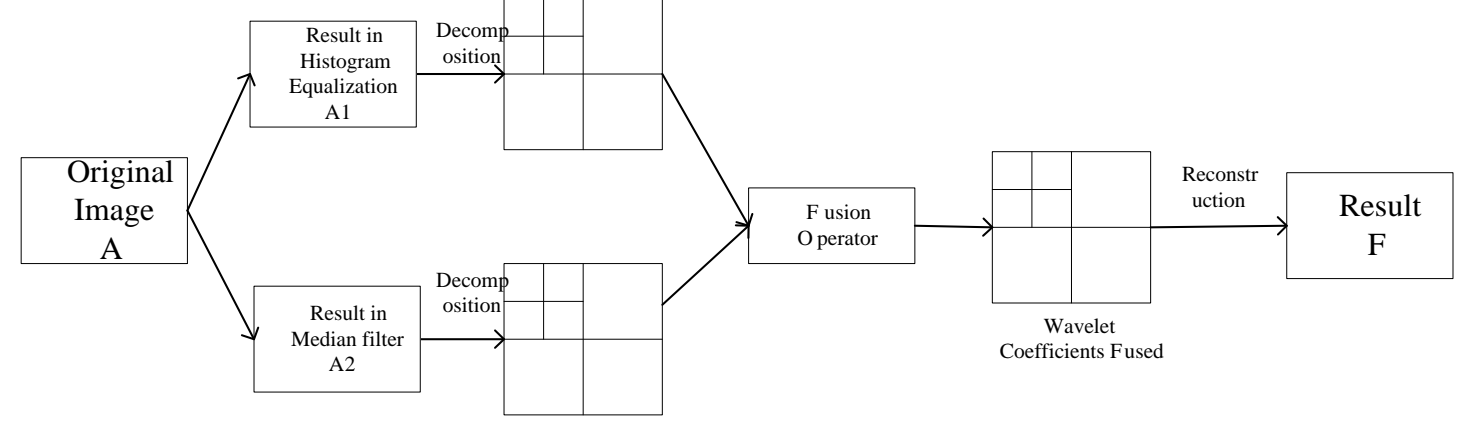

Fig. 1 the wavelets transform-based image fusion

The basic steps of fusion are as follows:

/* Algorithm 1----Image Enhancement Based on Wavelet Fusion */

(1) Histogram equalization is applied to breast $\mathrm{X}$-ray image $A$, resulting in figure $A 1$, which can increase the dynamic range of the pixel gray values and improve the overall image contrast.

(2) Median Filter is applied to the breast X-ray image $A$, resulting in figure $A 2$, which reduces the image noise and keeps the information with outline of image.

(3) Figures $A 1$ and $A 2$ are decomposed respectively into two levels by multi-wavelet transform which selects the appropriate wavelet basis function.

(4) According to the low-frequency and high-frequency characteristics, the coefficients for multi-wavelet decomposition are processed as the following strategy: adopt the higher energy for an efficient fusion method to low-frequency and the bigger variance to high-frequency.

(5) With the low-frequency component and high-frequency component, the higher quality image $F$ is get by reconstructing with inversing wavelet transform.

Breast image segmentation. A new breast image segment method is proposed. Firstly, Algorithm 1 is applied to pretreated images. Secondly, an improved live-wire interactive segmentation algorithm with qualification to make the shortest path search is used to divide suspicious masses on enhanced images. The new method is described as follows:

/* Algorithm 2----Breast image segmentation */

(1) Enhance an image by algorithm 1.

(2) Calculate gradient magnitude $f_{G}$, gradient direction $f_{D}$, and Canny operator $f_{C}$ for an image, while calculating cost of empowering figure $Q$ which is based on cost function $l_{\text {new }}(p, q)$.

(3) Initialize search node set $V$ which is set as $\{a\}$, cumulative weight matrix $D$ as $+\infty$, the path marked matrix $P$ as 0 . And mark the path matrix as $\{1,2,3,4\}$ to represent its four areas of nodes.

(4) Remove node $i$ from set $V$, which is the smallest cumulative path;

(5) Read distance's weights $d_{i j}$, which is the distance's weight for node $i$ to its four directions around 4 areas . Compare $D_{j}$ and $D_{i}+d_{i j}$. If $D_{j}<D_{i}+d_{i j}$, replace the value of $D_{j}$ with $D i+d_{i j}$, modify the path matrix values of corresponding directions, and put the node into search set $V$. 
(6) If target point $a$ is not a 4-point field node $i$, then turn to (3); otherwise turn to (7);

(7) According to path matrix, the shortest path between $p$ and $q$ is found by tracking its direction information starting from the direction of the target node.

\section{Experimental Results and Analysis}

We did experiments to check the effect of the improved wavelet-based fusion algorithm, which were executed on a computer with Pentium Dual-Core E5300, 2.6GHz, 2G memories, Matlab7.0.

Image enhancement. 150 experimental breast X-ray images selected from the Mammographic Image Analysis Society, MIAS ${ }^{[12]}$ and 10 clinical breast X-ray images from a hospital in China were used in the first experiment to compare the Histogram equalization and Median filtering with our method, Algorithm1.

Enhancement processes were done in original typical breast X-ray image with the histogram equalization, Median filtering, Algorithm1 separately. And the results are shown in Fig.2.

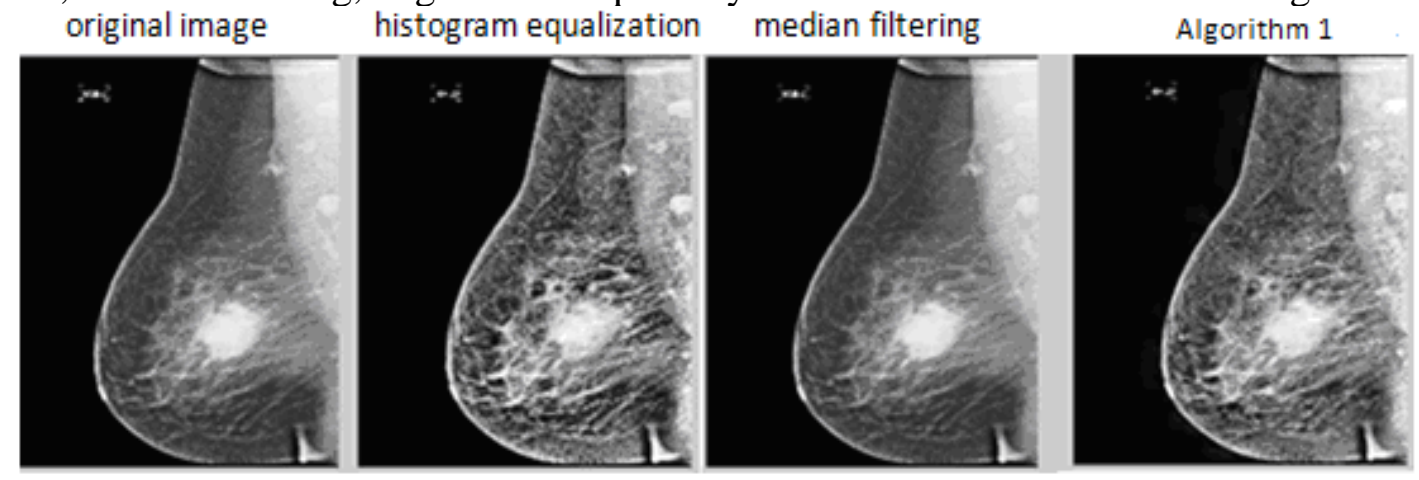

Fig. 2 The results of several image enhancement algorithms.

Another experiment was to enhance ROI with a size of $200 * 200$, and the results are shown in Fig. 3. original image

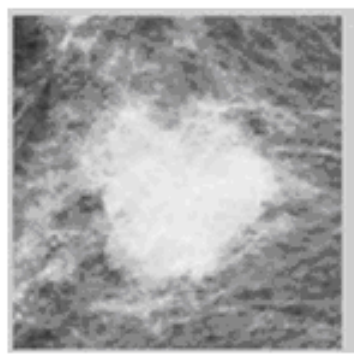

histogram equalization

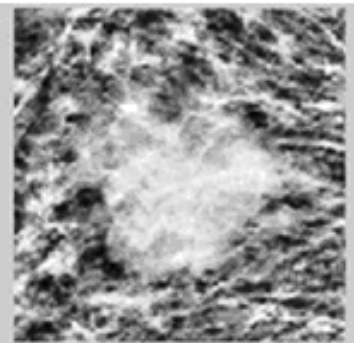

median filtering

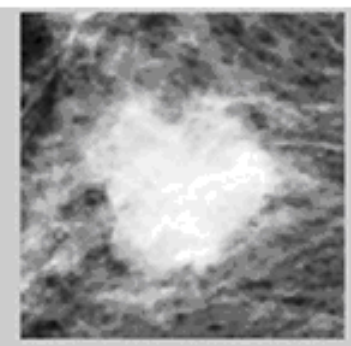

Algorithm 1

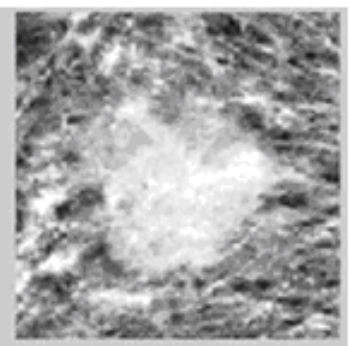

Fig.3 The results of several image enhancement algorithms

In Fig. 2 and Fig. 3, the edge of the outline is relatively clear after the use of histogram equalization method, but the breast X-ray image has noise because of its more glandular tissues. With median filtering, the breast lumps region is obviously enhanced, and a lot of noise reduced, but the bright details are weakened. With Algorithm1, the noise is effectively eliminated, and the breast lumps areas are more obvious, and breast edge is also enhanced.

In order to quantitatively compare pretreatment effect of image, objective evaluation criteria, such as information entropy, average gradient and contrast improvement index $C I I$, are introduced to evaluate pretreatment effect in this paper.

Information entropy is one of the most important features of image, which reflects the details of image. The larger information entropy, the more abundant image details. Information entropy is defined as Eq.11.

$$
E=-\sum_{i=0}^{255} P_{i} \log _{2} P_{i}
$$


Where $P_{i}$ is the probability of gray level $i$.

Average gradient also is an important feature of image, which reflects the ability of image on the expression of detail contrast, and is used to evaluate the clarity of image. Average gradient reflects the characteristics of image detail variance degree and texture changes on the whole. The greater general average gradient, the clearer image. Average gradient is defined as Eq.12.

$$
A=\frac{1}{(M-1)(N-1)} \sum_{i}^{M-1} \sum_{j}^{N-1} \sqrt{\left(\left(\frac{\partial f\left(x_{i}, y_{j}\right)}{4 \partial x_{i}}\right)^{2}+\left(\frac{\partial f\left(x_{i}, y_{j}\right)}{4 \partial y_{i}}\right)^{2}\right)}
$$

Where $M, N$ are respectively the rows and columns of image.

Contrast improvement index CII reflects image contrast. $C I I$ is defined as Eq.13.

$$
\mathrm{CII}=\frac{\mathrm{C}_{\text {processed }}}{\mathrm{C}_{\text {original }}}
$$

An image is divided into small images of $5 \times 5, C$ is the average value of all contrast. The contrast of small image is defined as $\frac{\text { Max - Min }}{M a x+M i n}$ (Max is the block image gray value of the larger value, and Min is the block image gray value of the smaller).

Table 1. The comparison of image statistical parameters corresponding to Fig.2

\begin{tabular}{lccc}
\hline Evaluation parameters & $\begin{array}{l}\text { information } \\
\text { entropy }\end{array}$ & average gradient & CII \\
\hline Original image & 5.2698 & 0.0082 & - \\
Histogram equalization & 5.6665 & 0.0155 & 1.0681 \\
The median filter & 5.4541 & 0.0068 & 0.9901 \\
Algorithm 1 & 6.9639 & 0.0189 & 1.3025 \\
\hline
\end{tabular}

Table 2.The comparison of image statistical parameters corresponding to Fig.3

\begin{tabular}{lccc}
\hline Evaluation parameters & information entropy & Average gradient & CII \\
\hline Original image & 5.3326 & 0.0089 & - \\
Histogram equalization & 5.4746 & 0.0161 & 0.9912 \\
The median filter & 5.3205 & 0.0073 & 1.0012 \\
Algorithm 1 & 7.2293 & 0.019 & 1.4031 \\
\hline
\end{tabular}

Objective performance evaluation of two breast X-ray images is given by table 1 and table 2 . The results show that Algorithm1 comes to the biggest value of information entropy, average gradient, CII value among all algorithms in table 1 and table 2 . Algorithm 1 effectively increases in contrast, and eliminates noise while preserving edge details, and improves image quality.

Image segmentation. This section is to do simulation experiments on breast X-ray images with the segmentation method proposed in the last section, Algorithm 2. 


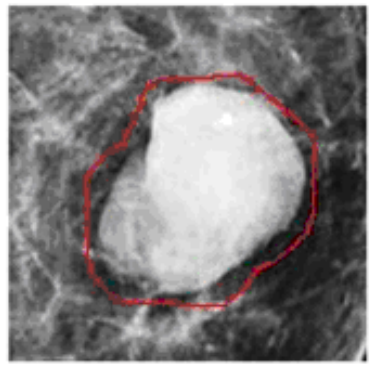

(a) doctors circle with original image

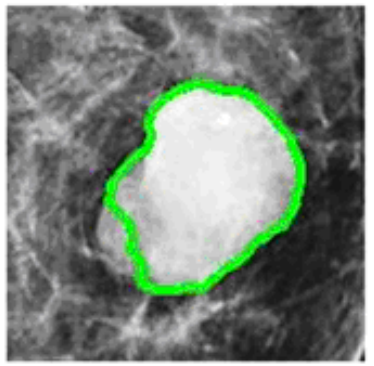

(b) segmentation with original image

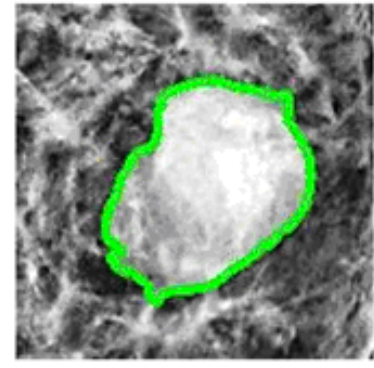

(c) segmentation with image enhanced Algorithm 2

Fig.4 Image Segmentation for typical breast mass

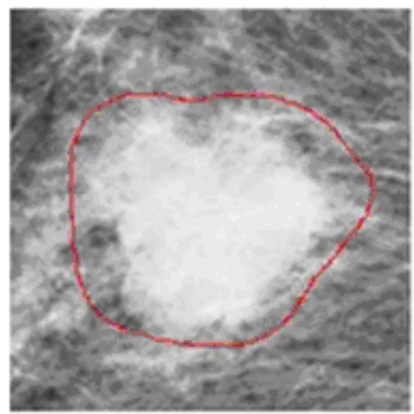

(a) doctors circle with original image

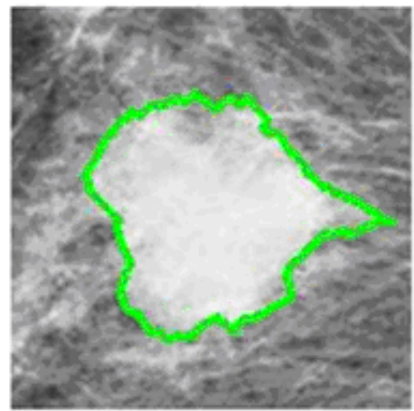

(b) segmentation with original image

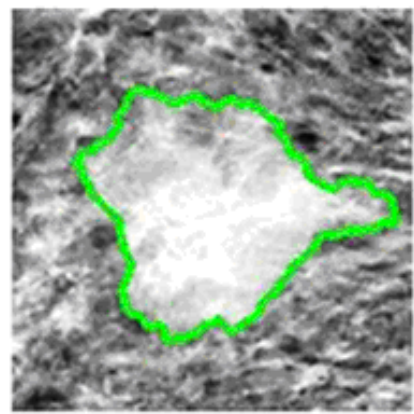

(c) segmentation with image enhanced Algorithm 2

Fig. 5 Image Segmentation for breast mass with burr

A group of images with typical breast lumps was selected to do comparing experiment. In one experiment, the improved live-wire algorithm was used to segment directly the original images. In another, the improved live-wire algorithm was used to segment an image enhanced by Algorithm 1 . The results are shown in Fig.4. Fig.4(a) is the circle painted by doctors to indicate lumps raw edge region of interest. Fig.4 (b) is the result of segmenting on an original image with the method of Paper [10]. Fig.4 (c) is the result of segmentation with Algorithm 2.

Fig. 5 shows the results of segmentation processing for breast mass with burr. Fig.5 (a) is the original area of interest for the circle painted by doctors indicating lumps edge. Fig.5 (b) is the result segmented by the method of Paper [10]. Fig.5 (c) is the result segmented by our method, Algorithm 2.

The results show that for a variety of typical breast mass, segmenting the interest region on enhanced image is better than on the original image

Running time comparison. X-ray images of breast lumps with different resolutions $150 * 150$, $200 * 200$, or $300 * 300$ were segmented with traditional live-wire algorithm[10] and Algorithm 2 separately, and five images were selected for each size. The results are shown in Table 3.

Table 3 Comparison of traditional live-wire algorithm and Algorithm 2

\begin{tabular}{cccc}
\hline Image Size & $150 * 150$ & $200 * 200$ & $300 * 300$ \\
\hline $\mathrm{T} 1$ (sec) & 13.093 & 34.5 & 50.23 \\
$\mathrm{~T} 2$ ( $\mathrm{sec})$ & 0.179 & 1.007 & 1.41 \\
\hline
\end{tabular}

In table 3, $T 1$ is the average time which the traditional live-wire algorithm takes. $T 2$ is the average time Algorithm 2 takes. As can be seen from the experimental data, Algorithm 2 can effectively improve operational efficiency.

\section{Conclusion}

In this paper, an algorithm based on wavelet fusion for mammogram image enhancement and segmentation was presented. Firstly, based on the image preprocessing with histogram equalization method and median filter, an efficient wavelet fusion method was proposed to realize the 
enhancement for mammography image by adopting the fusion rule of taking large low frequency energy and high-frequency variance. Secondly, an improved live-wire interactive segmentation algorithm was used to do edge division of suspicious masses, which qualification was set to make the shortest path searching more effective. The experimental results indicate that Algorithm 2 can extract edge more accurately and get better segmentation for breast X-ray masses than method of Paper [10]. Further work is needed to explore automatic capabilities of segmentation algorithm.

\section{References}

[1]Pereira, DC ; Ramos, RP ; do Nascimento, MZ. Segmentation and detection of breast cancer in mammograms combining wavelet analysis and genetic algorithm. COMPUTER METHODS AND PROGRAMS IN BIOMEDICINE , 2014, Vol. 114 (2014),No.1,p.88

[2] Tie-jun Yang, Xiao-ming Wu, Lin Huang. Method of Breast Lumps Segmentation Based on Immune Algorithm and Level Set. Computer Simulation, 2010, No.3, p.243

[3] LI Yan-Feng, CHEN Hou-Jin, YANG Na, ZHANG Sheng-Jun. Pectoral Muscle Segmentation in Mammograms Based on Anatomic Features . Acta Automatica Sinica, Vol. 39(2013), No.8, p.1265

[4] Cao A Z, Song Q, Yang X L, et al. Breast mass segmentation on digital mammograms by a combined deterministic annealing method. Biomedical Imaging: Nano to Macro, 2004. IEEE International Symposium on. IEEE, 2004, p.1303

[5] Amutha, S., Babu, D.R.R., Shankar, M.R., Kumar, N.H. Mammographic image enhancement using modified mathematical morphology and Bi-orthogonal wavelet. 2011 International Symposium on, 2011, p.548

[6] Aydin A, et al. A wavelet neural network for the detection of microcalcifications in multiscale enhanced digitized mammograms. IEEE, vol. 1(1999), p.389

[7] LUAN Meng-jie, WEN Xue-bing. Mammogram image enhancement based on wavelet fusion. Computer Engineering and Applications, Vol. 46(2010), No.18, p.177

[8] Sakellaropoulos P, Costaridou L and Panayiotakis G. A wavelet-based spatially adaptive method for mammographic contrast enhancement [J]. Phys. Med. Biol., vol.48 (2003), p.787

[9] Barrett W., Mortensen E.. Interactive live-wire boundary extraction . Medical Image Analysis. Vol. 1(1997), No. 4, p.331

[10] DANG Jian-wu, ZHANG Fang, HU Tie-jun, CHAO Ying. Research and Improvement of Live-Wire Interactive Algorithm for Medical Image Segmentation. Application Research of Computers, Vol 25(2008), No. 10, p.3048

[11] WANG Yang-ping, DANG Jian-wu, LI Qiang,et al. Live-wire Algorithm for Medical Image Segmentation based on Improved Search Strategy. Computer Engineering and Applications, Vol. 43(2007), No. 29, p.24

[12] Information on http://www.mammoimage.org/databases/ 